\title{
Mouse Embryonic Tooth Germ Dissection and Ex vivo Culture Protocol \\ Xue Han ${ }^{1, \#}$, Keigo Yoshizaki ${ }^{1, \#, ~ *, ~ T i a n ~ T i a n ~}{ }^{1}$, Kanako Miyazaki $^{1}$, Ichiro Takahashi ${ }^{1}$ and Satoshi Fukumoto ${ }^{2,3, *}$
}

\begin{abstract}
${ }^{1}$ Section of Orthodontics and Dentofacial Orthopedics, Division of Oral Health, Growth and Development, Kyushu University Faculty of Dental Science, Fukuoka, Japan; 'Division of Pediatric Dentistry, Department of Oral Health and Development Sciences, Tohoku University Graduate School of Dentistry, Sendai, Japan; ${ }^{3}$ Section of Pediatric dentistry, Division of Oral Health, Growth and Development, Kyushu University Faculty of Dental Science, Fukuoka, Japan

*For correspondence: yosizaki@dent.kyushu-u.ac.jp; fukumoto@dent.tohoku.ac.jp

\#Contributed equally to this work
\end{abstract}

\begin{abstract}
[Abstract] A tooth germ ex vivo organ culture allows visualization of its development in different stages, thus enabling investigation of the molecular mechanisms of regulatory factors. Tooth germs can be rapidly dissected from E13 mouse embryos and placed on cell culture inserts for observation of subsequent tooth germ development in a three-dimensional situation in real time. This method is also suitable for other organs that develop by epithelial-mesenchymal interactions, including salivary gland, hair, lung, and kidney. In addition, siRNAs or growth factors can be easily added to ex vivo tooth germ cultures to investigate the detailed molecular function of specific genes. The present protocol provides an efficient and practical method for isolation and ex vivo culture of embryonic tooth germs.
\end{abstract}

Keywords: Organ culture, Embryonic tooth germ isolation, Ex vivo culture, Tooth development, Organogenesis, Epithelial-mesenchymal interaction

[Background] Teeth are considered to represent a typical epithelial-mesenchymal organ. When investigating tooth development, observation of the developmental pattern can help to elucidate the mechanisms of morphogenesis biology. When transfecting siRNAs or expression vectors into embryonic organs, a maternal injection protocol is commonly used in studies of mice. However, with traditional methods, it is difficult to efficiently transport reagents into the target organ. On the other hand, an ex vivo organ culture simplifies direct transportation of reagents into a dissected organ, allowing for observations in a three-dimensional manner. In addition to teeth, other epithelial-mesenchymal organs such as salivary gland and hair can also be successfully cultured with this system.

Several different organ culture protocols have been presented for tooth germ organ culturing, such as a semi-solid method (Heywood and Appleton, 1984), with Matrigel (Shih and Sander, 2014; Sun et al., 2016), and with the use of mesh placed in cell culture dishes (Yamada et al., 1980; Bringas et al., 1987; Tabata et al., 1996; Fukumoto et al., 2006; Yamamoto et al., 2008; Yamada et al., 2016). However, each requires preparation of the culture materials in the wells every time prior to organ dissection. In contrast, with the present organ culture system, the materials can be directly purchased from a supplier, which saves time and avoids inevitable contamination during preparation of the culture materials. 
Traditional methods used to examine the functions of gene or drugs in vivo require time to determine the results individually, whereas with an ex vivo culture, it is possible to examine a large number of organ samples at the same time. Furthermore, it is possible to screen for critical genes or other related factors during organ development.

Here, we describe a relatively simple and efficient method for ex vivo organ culture of embryonic tooth germs. With the use of this protocol, screening studies and development observations can be objectively and directly conducted.

\section{Materials and Reagents}

1. $100 \mathrm{~mm}$ cell culture dish (Falcon, catalog number: 353003 )

2. $60 \mathrm{~mm}$ cell culture dish (Falcon, catalog number: 353002 )

3. Cell culture 6-well plate for insert (Falcon, catalog number: 353502)

4. Cell culture insert ( 6 wells, $8 \mu \mathrm{m}$, Falcon, catalog number: 353093 )

5. Thirteen-day-pregnant female mouse

6. Fetal bovine serum (FBS, Gibco, catalog number: 10437028), short term storage at $4{ }^{\circ} \mathrm{C}$, long term storage at $-20^{\circ} \mathrm{C}$, heat inactivation not necessary

7. siRNA: Nkx2-3 (Dharmacon, ON-TARGET Plus L-057189-01-0002), storage at $-20{ }^{\circ} \mathrm{C}$; control (Dharmacon, ON- TARGET Plus Nontargeting Control Pool D-001810-05), storage at $-20^{\circ} \mathrm{C}$

8. $70 \%$ ethanol

9. Dulbecco's phosphate buffered saline without $\mathrm{Ca}^{2+}$ or $\mathrm{Mg}^{2+}$ (D-PBS(-), Wako, catalog number: 04529795), storage at room temperature

10. Dulbecco's modified Eagle's medium (DMEM)/F-12 (Gibco, catalog number: 11330032), storage at $4{ }^{\circ} \mathrm{C}$

11. L-Glutamine (200 mM, 100x) (Gibco, catalog number: 25030081$)$, storage at $-20^{\circ} \mathrm{C}$

12. Penicillin-streptomycin (Liquid, Gibco, catalog number: 15140122 ), storage at $-20^{\circ} \mathrm{C}$

13. L-Ascorbic acid (Sigma-Aldrich, catalog number: A4544-25G), storage at room temperature

14. Lipofectamine 3000 with Plus reagent (Life Technologies, catalog number: L3000-015), storage at $4{ }^{\circ} \mathrm{C}$

15. Opti-MEM (Gibco, catalog number: 31985062 ), storage at $4{ }^{\circ} \mathrm{C}$

16. Working medium (see Recipes)

17. Organ culture medium (see Recipes)

18. siRNA transfection medium (see Recipes)

\section{Equipment}

1. Surgical instruments: tweezers, scissors, fine scissors, precision tweezers (Dumont \#5 Forceps) (Figure 1A)

2. Compact Greenough Stereo Microscope (ZEISS, model: Stemi 305 EDU) (Figure 1B) 
3. Mouse dissection table

4. $\mathrm{CO}_{2}$ incubator (ASTEC, model: SCA-80DS)

5. Research inverted system microscope (Olympus, model: IX71)

A

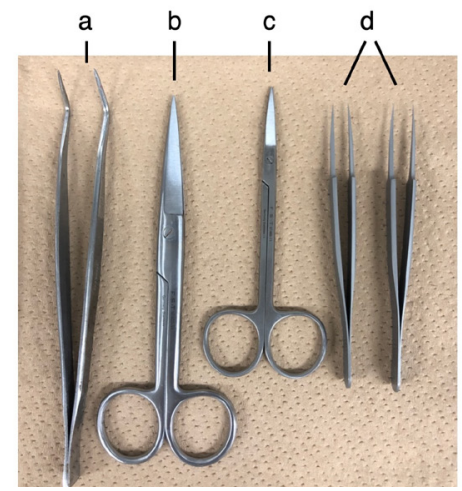

B

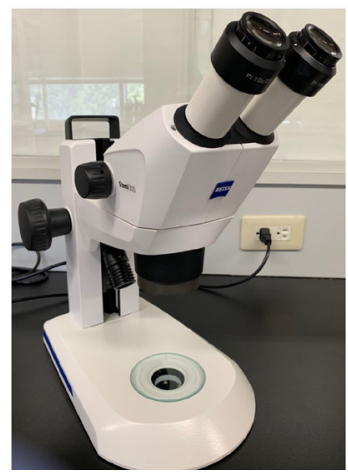

Figure 1. Instruments used for embryonic mandible isolation and molar germ dissection. $A$. Surgical instruments: a. tweezers, b. scissors, c. fine scissors, d. precision tweezers. B. Compact Greenough stereo microscope.

\section{Procedure}

A. Preparation

1. Prepare two $100 \mathrm{~mm}$ cell culture dishes and add approximately $20 \mathrm{ml}$ of D-PBS to each, also two $60 \mathrm{~mm}$ cell culture dishes and add approximately $6 \mathrm{ml}$ of D-PBS to each. Then, place four dishes on ice.

2. Prepare working medium (follow Recipe 1, keep on ice), with $50 \mathrm{ml}$ of working medium enough for 20 embryos.

3. Prepare two $60 \mathrm{~mm}$ cell culture dishes and add approximately $6 \mathrm{ml}$ of working medium, then place on ice.

4. Prepare organ culture medium (follow Recipe 2, keep at $4{ }^{\circ} \mathrm{C}$ ), use $1.8 \mathrm{ml}$ of organ culture medium for each well. 
B. Embryonic mandible isolation (Figure 2)

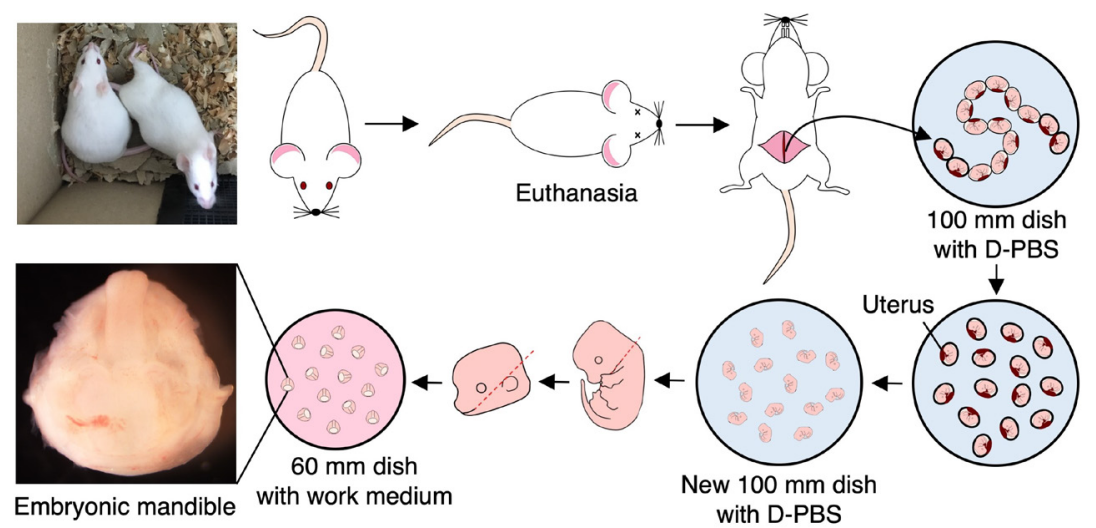

Figure 2. Procedure for isolation of embryonic mandible

1. Euthanize 13-day-pregnant female mouse according to local guidelines. Place the mouse on back on dissection table.

2. Soak mouse abdomen fur with $70 \%$ ethanol.

3. Cut skin with tweezers (Figure 1A-a) and scissors (Figure 1A-b), then pull away skin to expose abdominal wall.

4. Cut abdominal wall with tweezers (Figure 1A-a) and fine scissors (Figure 1A-c).

5. Use tweezers (Figure 1A-a) to lift up the uterine horns and cut them away with fine scissors (Figure 1A-c). Place in $100 \mathrm{~mm}$ dish with cold D-PBS on ice (Figure 2).

6. Separate uterus in D-PBS dish with cleaned scissors.

7. Isolate each embryo by gently slicing through uterus with precision tweezers (Figure 1A-d).

8. Transfer embryos to new D-PBS dish.

9. Separate head and body parts of embryos with precision tweezers under stereo microscope (Figure 1B) in $60 \mathrm{~mm}$ D-PBS dish.

10. Cut off mandible through line from corner of mouth to ear (Figure 2). Next, move separated mandibles into new $60 \mathrm{~mm}$ D-PBS dish on ice (Video 1). 


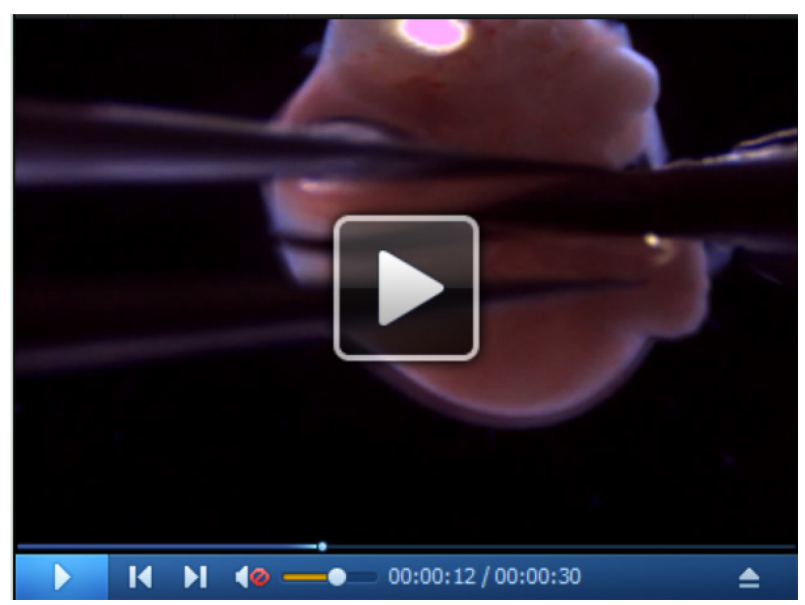

Video 1. Mandible separation

C. Mandibular molar germ dissection and seeding

1. Transfer mandible into $60 \mathrm{~mm}$ dish containing working medium under stereo microscope.

2. The mandible molars can be seen on the side of the tongue (Figure $3 \mathrm{~A}$ ). Carefully separate tooth germ including surrounding mesenchyme from the mandible with precision tweezers, and place them into new $60 \mathrm{~mm}$ dish containing working medium on ice (Figure 3B).

3. Add $1.8 \mathrm{ml}$ of organ culture medium to each well of 6-well plate, then place cell culture inserts into wells (Figures 3C-3D).

4. Place tooth germs on inserts with epithelium down and mesenchyme up, which allows for epithelium side to be easily seen under microscope. When tooth germ is placed in opposite manner, observation of tooth shape is difficult. Epithelium looks hyaline and mesenchyme contains capillary vessels can be seen. Approximately 9 tooth germs can be seeded in 1 well (Figure 3D).

5. Culture tooth germs at $37{ }^{\circ} \mathrm{C}$ in a humidified atmosphere of $5 \% \mathrm{CO}_{2}$.

6. Take photos under microscope every 2 or 3 days. Representative developing tooth, salivary gland, and hair are shown in Figure 4.

7. Organ culture medium should be changed every 3 days. 
A

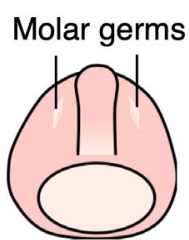

C
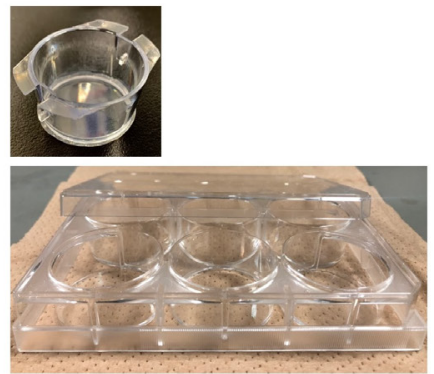

B
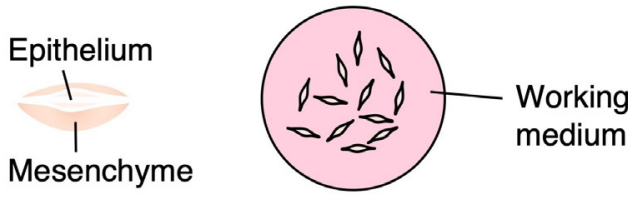

D
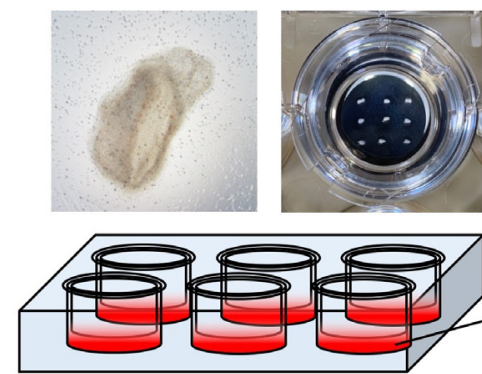

Organ

culture

medium

Figure 3. Procedure for mandibular molar germ dissection. A. Embryonic mandible. B. Tooth germs. C. Organ culture plates. D. Tooth germs in plates.

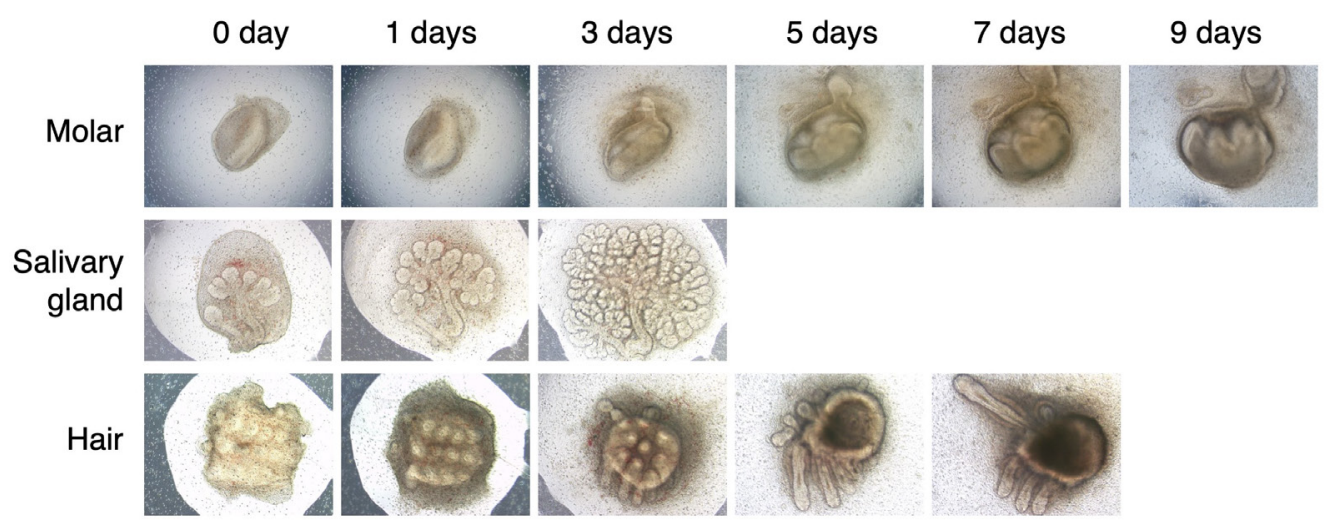

Figure 4. Development of epithelial-mesenchymal tissue using ex vivo organ culture

D. siRNA-mediated knockdown method

1. Prepare siRNA transfection mixture with $500 \mathrm{nM}$ of siRNA using Lipofectamine 3000 reagent according to manufacturer's protocol (follow Recipe 3).

2. Drop $8 \mu \mathrm{l}$ of siRNA transfection mixture directly on top of each tooth germ. Culture tooth germs at $37^{\circ} \mathrm{C}$ in humidified atmosphere of $5 \% \mathrm{CO}_{2}$ for 2 to 7 days. siRNA must be added every 2 or 3 days.

3. For observations, take photos under microscope every 2 or 3 days (Figure 5). 


\section{E 13.5 + 7- day culture}
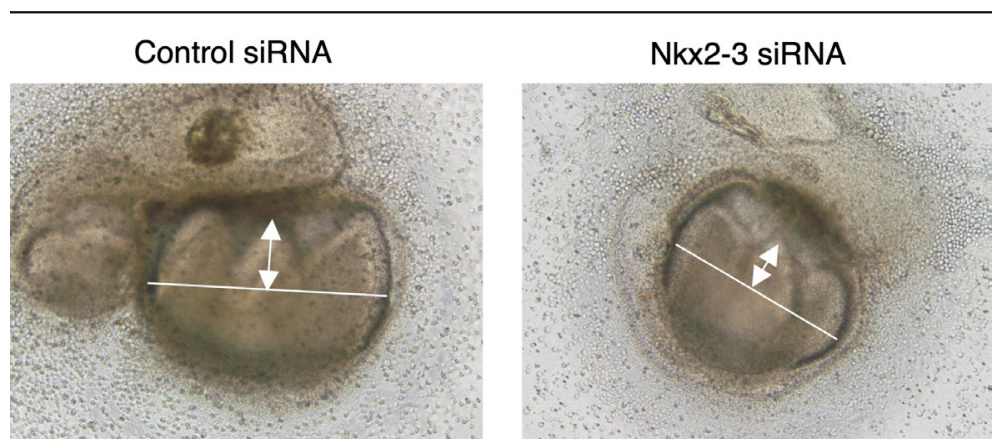

Figure 5. Morphological changes by addition of Nkx2-3 siRNA

\section{Data analysis}

With the present protocol, approximately 24 tooth germs can be dissected from a single E13 fetal ICR mouse. Tooth, salivary gland, and hair development can be observed from 1 day to a maximum of 2 weeks in the same medium and culture conditions (Figure 4). The sizes and shapes of the organs can be analyzed using the ImageJ software package (Wayne Rasband, National Institutes of Health). mRNA and protein expression can also be analyzed by use of a traditional RNA and protein isolation method.

\section{$\underline{\text { Notes }}$}

1. Euthanasia should be performed according to local guidelines.

2. Tweezers and scissors should be cleaned by wiping with $70 \%$ ethanol between uses.

3. To avoid bacterial contamination, do not allow fur to come into contact with uterus when removing from abdomen.

4. Separate mandible and maxilla through line between eye and ear to avoid damage to mandibular tooth germs.

5. Frequently change to clean D-PBS or work medium during dissection.

6. Be careful to not touch the membrane of the insert with forceps tip during tooth germ seeding.

7. Make sure tooth germs are not dipped into organ culture medium during long-term culture period.

8. Cell culture inserts with a $0.4-\mu \mathrm{m}$ pore size can also be used in this system.

\section{$\underline{\text { Recipes }}$}

1. Working medium

DMEM/F-12 supplemented with:

$1 \%$ penicillin-streptomycin

2. Organ culture medium

DMEM/F-12 supplemented with: 
$20 \%$ fetal bovine serum

$180 \mu \mathrm{g} / \mathrm{ml}$ ascorbic acid

$2 \mathrm{mM}$ L-glutamine

$1 \%$ penicillin-streptomycin

3. siRNA transfection medium (for 10 tooth germs)

a. Opti-MEM, $50 \mu \mathrm{l}+$ Lipofectamine $3000,6 \mu \mathrm{l}$

b. Opti-MEM, 50ul + siRNA, $50 \mathrm{pmol}$

Mix together and incubate mixture for 5 min at room temperature

Apply $8 \mu$ of mixture to each tooth germ

\section{Acknowledgments}

This work was supported in part by a grants-in-aid for Scientific Research (18H03012, 17K19765, $15 \mathrm{H} 05688$ to K. Y.; $16 \mathrm{H} 07065$ to K. M. and $17 \mathrm{H} 01606$ to S. F.). This protocol was originally published in J Biol Chem (Han et al. 2018).

\section{Competing interests}

The authors have no financial or research related conflicts of interest to declare.

\section{Ethics}

All animal experiments were approved by the ethics committee of the Kyushu University Animal Experiment Center (protocol no. A26-208-0, April 1st 2018- Match 31th 2020).

\section{References}

1. Bringas, P., Jr., Nakamura, M., Nakamura, E., Evans, J. and Slavkin, H. C. (1987). Ultrastructural analysis of enamel formation during in vitro development using chemicallydefined medium. Scanning Microsc 1(3): 1103-1108.

2. Fukumoto, S., Miner, J. H., Ida, H., Fukumoto, E., Yuasa, K., Miyazaki, H., Hoffman, M. P. and Yamada, Y. (2006). Laminin alpha5 is required for dental epithelium growth and polarity and the development of tooth bud and shape. J Biol Chem 281(8): 5008-5016.

3. Han, X., Yoshizaki, K., Miyazaki, K., Arai, C., Funada, K., Yuta, T., Tian, T., Chiba, Y., Saito, K., Iwamoto, T., Yamada, A., Takahashi, I. and Fukumoto, S. (2018). The transcription factor NKX23 mediates p21 expression and ectodysplasin-A signaling in the enamel knot for cusp formation in tooth development. J Biol Chem 293(38): 14572-14584.

4. Heywood, B. R. and Appleton, J. (1984). The ultrastructure of the rat incisor odontoblast in organ culture. Arch Oral Biol 29(4): 327-329. 
5. Shih, H. P. and Sander, M. (2014). Pancreas development ex vivo: culturing embryonic pancreas explants on permeable culture inserts, with fibronectin-coated glass microwells, or embedded in three-dimensional Matrigel ${ }^{\mathrm{TM}}$. Methods Mol Biol 1210: 229-237.

6. Sun, G., Liu, W., Fan, Z., Zhang, D., Han, Y., Xu, L., Qi, J., Zhang, S., Gao, B. T., Bai, X., Li, J., Chai, R. and Wang, H. (2016). The three-dimensional culture system with matrigel and neurotrophic factors preserves the structure and function of spiral ganglion neuron in vitro. Neural Plast 2016: 4280407.

7. Tabata, M. J., Kim, K., Liu, J. G., Yamashita, K., Matsumura, T., Kato, J., Iwamoto, M., Wakisaka, S., Matsumoto, K., Nakamura, T., Kumegawa, M. and Kurisu, K. (1996). Hepatocyte growth factor is involved in the morphogenesis of tooth germ in murine molars. Development 122(4): 1243-1251.

8. Yamada, M., Bringas, P., Jr., Grodin, M., MacDougall, M., Cummings, E., Grimmett, J., Weliky, B. and Slavkin, H. C. (1980). Chemically-defined organ culture of embryonic mouse tooth organs: morphogenesis, dentinogenesis and amelogenesis. J Biol Buccale 8(2): 127-139.

9. Yamada, A., Futagi, M., Fukumoto, E., Saito, K., Yoshizaki, K., Ishikawa, M., Arakaki, M., Hino, R., Sugawara, Y., Ishikawa, M., Naruse, M., Miyazaki, K., Nakamura, T. and Fukumoto, S. (2016). Connexin 43 is necessary for salivary gland branching morphogenesis and FGF10induced ERK1/2 phosphorylation. J Biol Chem 291(2): 904-912.

10. Yamamoto, S., Fukumoto, E., Yoshizaki, K., Iwamoto, T., Yamada, A., Tanaka, K., Suzuki, H., Aizawa, S., Arakaki, M., Yuasa, K., Oka, K., Chai, Y., Nonaka, K. and Fukumoto, S. (2008). Platelet-derived growth factor receptor regulates salivary gland morphogenesis via fibroblast growth factor expression. J Biol Chem 283(34): 23139-23149. 